\title{
Muséologies
}

Les cahiers d'études supérieures

muséologies

\section{Regarder et lire}

\section{Réflexion sur le rôle du texte dans une exposition d'art}

\section{Alice Chatzimanassis}

Volume 6, numéro 2, 2013

URI : https://id.erudit.org/iderudit/1018931ar

DOI : https://doi.org/10.7202/1018931ar

Aller au sommaire du numéro

\section{Éditeur(s)}

Association Québécoise de Promotion des Recherches Étudiantes en Muséologie (AQPREM)

\section{ISSN}

1718-5181 (imprimé)

1929-7815 (numérique)

Découvrir la revue

\section{Citer cet article}

Chatzimanassis, A. (2013). Regarder et lire : réflexion sur le rôle du texte dans une exposition d'art. Muséologies, 6(2), 117-131.

https://doi.org/10.7202/1018931ar

\section{Résumé de l'article}

Une évaluation a été menée en avril 2010 au musée d'Ixelles, à Bruxelles. Au moyen d'entretiens conduits à la sortie du musée, il s'agissait d'identifier et de comprendre les modes d'appropriation et le comportement des visiteurs envers le dispositif textuel dans deux expositions en cours, à savoir $E L$ CUBISMO. Cubism and its Context from the Fundación Telefónica Art Collection et Henri de Toulouse-Lautrec : La collection complète des affiches. Si l'importance du texte en tant qu'aide à l'interprétation dans les musées est aujourd'hui largement reconnue, l'enquête qualitative a permis de développer une réflexion sur les spécificités de l'écrit quand il est confronté à des oeuvres d'art et sur ses fonctions dans la pratique de contemplation et d'interprétation chez les visiteurs.
Tous droits réservés (C) Association Québécoise de Promotion des Recherches Étudiantes en Muséologie (AQPREM), 2013
Ce document est protégé par la loi sur le droit d'auteur. L'utilisation des services d'Érudit (y compris la reproduction) est assujettie à sa politique d'utilisation que vous pouvez consulter en ligne.

https://apropos.erudit.org/fr/usagers/politique-dutilisation/ 
Article cinq

\section{Regarder et lire}

Réflexion sur le rôle du texte dans

une exposition d'art

Alice Chatzimanassis 
Une évaluation a été menée en avril 2010 au musée d'Ixelles, à Bruxelles. Au moyen d'entretiens conduits à la sortie du musée, il s'agissait d'identifier et de comprendre les modes d'appropriation et le comportement des visiteurs envers le dispositif textuel dans deux expositions en cours, à savoir EL CUBISMO. Cubism and its Context from the Fundación Telefónica Art Collection et Henri de Toulouse-Lautrec: La collection complète des affiches. Si l'importance du texte en tant qu'aide à l'interprétation dans les musées est aujourd'hui largement reconnue, l'enquête qualitative a permis de développer une réflexion sur les spécificités de l'écrit quand il est confronté à des ouvres d'art et sur ses fonctions dans la pratique de contemplation et d'interprétation chez les visiteurs.

Détentrice d'une maîtrise en histoire de l'art de l'Université libre de Bruxelles (2007), Alice Chatzimanassis est également titulaire, depuis 2010, d'une maîtrise en muséologie de I'Université du Québec à Montréal. Dans ce contexte, elle a effectué un stage au musée d'Ixelles, à Bruxelles, et renouvelé son expérience auprès de cette institution en y réalisant une évaluation muséale pour son travail dirigé de maîtrise. Cette première expérience de terrain l'a amenée à s'engager, dès septembre 2011, dans le doctorat international en muséologie, médiation et patrimoine (UQAM/Université d'Avignon et des Pays de Vaucluse). chatzimanassis.alice@courrier.uqam.ca 
Les recherches qui ont souligné et analysé l'apport du texte en tant qu'aide à l'interprétation dans les expositions et les musées sont aujourd'hui nombreuses ${ }^{1}$. Comme le rappellent Julie Desjardins et Daniel Jacobi, sous sa forme la plus élémentaire, l'écrit joue déjà un rôle fondamental par l'«interrelation " qui s'instaure entre l'objet et l'étiquette qui l'accompagne ${ }^{2}$ : celle-ci sert d'abord et avant tout à désigner, nommer et identifier. L'objet dépourvu de son étiquette dans la collection ou l'exposition perd aussitôt son identité, est "muet». Inversement, une étiquette n'a de sens que lorsqu'elle accompagne un objet. Par conséquent, l'étiquette constitue la première étape de la médiation entre l'objet exposé et le visiteur. La médiation s'entend ici comme "l'action visant à réconcilier ou mettre d'accord plusieurs parties et, dans le cadre du musée, le public du musée avec ce qui lui est donné à voir " ${ }^{3}$. À côté des cartels, les autres types d'écrits interviennent eux aussi dans la relation qui s'établit entre le visiteur et les œuvres. «Le va-et-vient de l'objet au texte permet au visiteur d'instruire un travail cognitif et/ou affectif comme identifier, interpréter, comprendre, confirmer une émotion, nommer ou ancrer cet item dans sa mémoire ${ }^{4}$."

Dans l'exposition d'art, le visiteur se partage entre la contemplation et la lecture. Le texte fait basculer le visiteur d'une activité émotionnelle et sensitive à une activité intellectuelle. Quelles sont alors les spécificités, ou, pour reprendre Serge Chaumier, l'" opérationnalité ${ }^{5}$ " du texte quand celui-ci est confronté à des œuvres d'art? La possible nuisance du texte à la contemplation des œuvres est régulièrement évoquée. «Les textes ne sont pas toujours bien acceptés. Pour certains, ils constituent une

1 À ce propos, voir notamment: GOTTESDIENER, Hana (dir.). Publics et Musées, $\mathrm{n}^{\circ}$ 1, 1992.

2 DESJARDINS, Julie et Daniel JACOBI. "Les étiquettes

dans les musées et les expositions scientifiques et techniques". Publics et Musées, $\mathrm{n}^{\circ} 1$ 1, 1992, p. 13-32.

3 MONTPETIT, Raymond. «Médiation ». In. DESVALLÉES, André et François MAIRESSE (dir.). Dictionnaire encyclopédique de muséologie. Paris: Armand Colin, 2011, p. 215. 4 DESJARDINS et JACOBI, «Les étiquettes dans les musées et les expositions...", op. cit., p. 15.

5 CHAUMIER, Serge. "Les méthodes de l'évaluation muséale. Quelques repères au sujet des formes et des techniques ». La Lettre de l'OCIM, nº 65, 1999, p. 16. pollution visuelle, une atteinte à l'esthétique de l'exposition et surtout ils détourneraient l'attention que les visiteurs doivent porter aux objets, aux œuvres d'art ${ }^{6}$." Dans le premier chapitre de L'Amour de l'art, intitulé «L'air du temps ", Pierre Bourdieu parle d'« idéologie charismatique " pour caractériser cette situation: conservateurs, amateurs d'art, professeurs et visiteurs habitués manifestent une certaine réticence à l'égard des panneaux écrits. La conception de l'art alors en vigueur veut que la délectation esthétique se passe de médiation, l'art ayant soi-disant le pouvoir d'émouvoir naturellement tout visiteur en vertu de sa grâce intrinsèque. Il serait donc inutile d'encombrer les œuvres avec des explications ${ }^{7}$. Une recherche conduite au musée du Louvre prouve cependant qu'«il n'y a pas compétition entre le temps de regard et le temps de lecture " ${ }^{8}$. Des expériences menées en psychologie de l'art ont également démontré que la perception des œuvres pouvait être modifiée par l'ajout d'information ${ }^{9}$. Le temps de regard accordé à une œuvre a par exemple parfois été augmenté par la présence d'un texte, ce temps de regard étant proportionnel à l'intérêt éprouvé pour l'œuvre ${ }^{10}$. La question est alors de savoir comment le texte peut améliorer la rencontre esthétique. Puisque les œuvres d'art ont été créées pour être vues (ce qui ne les empêche pas d'assurer d'autres fonctions comme le font un retable d'autel ou un portrait royal), le texte peut-il aider à mieux regarder? "On remarquera que le simple fait de lire ne suffit pas à augmenter l'attention, mais que la lecture peut, dans certaines conditions qui restent à déterminer, favoriser l'exploration visuelle de l'œuvre. Le contenu et la formulation de l'étiquette sont sans doute déterminants ${ }^{11}$. " À partir d'une étude portant sur les modalités de lecture des

6 GOTTESDIENER, Hana. "Introduction ». Publics et Musées, $\mathrm{n}^{\circ} 1,1992$, p. 11.

7 BOURDIEU, Pierre et Alain DARBEL. L'amour de l'art. Les musées d'art européen et leur public. Paris: Les Éditions de Minuit, 1969.

8 GOTTESDIENER, Hana. "La lecture de textes dans les musées d'art ". Publics et Musées, n 1, 1992, p. 86.

9 Pour une présentation de ces expériences, voir

GOTTESDIENER, ibid., p. 79.

10 Ibid., p. 80.

11 Ibid., p. 84. 
textes dans une exposition de peinture, c'est précisément du contenu des textes d'exposition que nous nous proposons de discuter dans les lignes qui suivent.

\section{Une évaluation des dispositifs textuels au musée d'Ixelles}

Sachant que le musée d'Ixelles envisage d'actualiser le dispositif didactique de ses collections permanentes, nous avons décidé de réaliser une évaluation des textes d'exposition dans le but de prendre connaissance des attentes du public et éventuellement de proposer, au regard de nos résultats, des pistes de travail pour le remaniement de ce dispositif. L'évaluation sommative ${ }^{12}$ doit nous permettre d'identifier le mode d'appropriation et le comportement des visiteurs envers les dispositifs textuels déployés au musée d'Ixelles. Les objectifs de l'enquête concernent divers aspects, à commencer par les modalités d'utilisation des textes ${ }^{13}$ : Les visiteurs les ont-ils repérés dans l'exposition? Les lisent-ils? Tous ou seulement quelques-uns? En entier ou partiellement? Nous voulons aussi mesurer la capacité didactique de ces textes: Sont-ils compris? Ont-ils transmis aux visiteurs des connaissances sur les artistes et les œuvres exposées? Qu'en retiennent les visiteurs? Il s'agit ensuite de prendre connaissance du degré d'appréciation de ces textes en recueillant les critiques des visiteurs quant à leur forme (apparence, situation dans l'exposition, fréquence) et à leur contenu (nature de l'information, apport de connaissances, niveau de langue). Enfin, nous souhaitons connaître les attentes des visiteurs du musée d'Ixelles à propos des textes

12 L'évaluation sommative s'effectue sur une exposition déjà ouverte au public. Elle permet aux concepteurs de rassembler, à partir de recherches conduites sur le terrain (par l'observation ou des entretiens), des informations sur la réception d'une exposition par les visiteurs, leurs réactions, attendues ou pas, face à certains éléments, ou encore sur le succès ou non des dispositifs de médiation. L'évaluation sommative intervient donc ponctuellement et sur différentes expositions. À ce propos, voir GOTTESDIENER, Hana.

Évaluer l'exposition. Définitions, méthodes et bibliographie sélective commentée d'études d'évaluation. Paris: La Documentation française, 1987, p. 9-13; CHAUMIER, Serge. «Les méthodes de l'évaluation muséale. Quelques repères d'exposition. L'enquête doit en somme nous amener à comprendre le rôle et l'influence des textes sur la pratique de contemplation des œuvres et la réception de l'exposition.

L'évaluation porte sur deux expositions qui ont eu lieu au même moment au musée d'Ixelles, à savoir EL CUBISMO. Cubism and its Context from the Fundación Telefónica Art Collection et Henri de Toulouse-Lautrec: La collection complète des affiches*. Les expositions temporaires du musée d'Ixelles se tiennent dans l'aile Morgan, un bâtiment rectangulaire de type halle, surplombé d'une verrière occultée. Il comprend un large espace au rez-de-chaussée, flanqué d'une scène, et deux galeries circulaires en balcon, la seconde étant depuis toujours fermée au public. L'exposition temporaire EL CUBISMO occupe le rez-de-chaussée, tandis que Lautrec occupe la première galerie. Il est intéressant de remarquer que cette architecture implique, pour la galerie circulaire, un parcours linéaire presque obligé, dans un sens ou dans un autre, mais forcément sans espace central de déambulation. Les affiches de Lautrec sont donc accrochées selon un parcours linéaire chronologique. Les deux expositions présentent des dispositifs textuels différents. Nous verrons cependant que des effets semblables ont été constatés dans les deux cas.

L'exposition EL CUBISMO a été commanditée et conçue par la Fundación Telefónica avec l'aide du musée d'Ixelles. Elle est divisée en trois grandes sections (voir plan en annexe): 1913-1940 Cubisme et modernité en Amérique latine: 1917-1925 La nouvelle vie du Cubisme et Géographie du Moderne (sur la scène). L'exposition rappelle, dès l'introduction,

au sujet des formes et des techniques ". La Lettre de l'OCIM, no 65, 1999, p. $13-17$.

13 Voici quelques questions issues du guide d'entretien: Avez-vous remarqué et lu les textes placés à ces endroits-ci? Avez-vous lu le texte d'introduction à l'exposition? Pourriez-vous m'indiquer sur le plan quel est le premier texte que vous avez lu après l'introduction? Vous souvenez-vous, brièvement, de ce dont il parle? Avez-vous le sentiment que les textes vous ont appris quelque chose? Lors de votre visite, avez-vous trouvé suffisante/satisfaisante l'information fournie par le musée concernant les œuvres et les artistes?

* (du 5 février au 25 avril 2010) 
la constitution de la collection Telefónica autour de l'œuvre du peintre cubiste espagnol Juan Gris. Elle présente également les œuvres d'artistes latino-américains influencés par Gris. La vie, l'œuvre et la place de l'artiste espagnol dans l'histoire de l'art moderne sont évoquées et resituées par rapport au cubisme de Braque et de Picasso. Le dispositif textuel comporte, sur le plan macro-structure ${ }^{14}$, le titre de l'exposition et le nom de la fondation, tous deux inscrits en très grandes lettres rouges sur fond noir. Un peu plus loin, le texte d'introduction, également en rouge sur noir, présente des caractéristiques formelles et typographiques propres, qui le différencient des autres textes d'information. Au niveau micro-structurel, toutes les œuvres de l'exposition EL CUBISMO sont accompagnées d'étiquettes autonymes ${ }^{15}$ comprenant les nom et prénom de l'artiste, les dates de naissance et de décès, le titre et la date de l'œuvre, ainsi que la technique. On dénombre ensuite onze textes plus importants dont la nature varie en fonction de leur emplacement et de leur contenu. Nous y reviendrons plus loin.

Si EL CUBISMO est une exposition temporaire, la collection complète des affiches de Lautrec, exposée en permanence, est au contraire le fleuron de la collection ixelloise. Le musée étant partiellement fermé en raison de rénovation des bâtiments, les affiches constituent, au moment de l'enquête, la seule partie des collections permanentes accessible au public, à côté de l'exposition sur le cubisme. Conscient de la richesse et de la qualité de ce fonds, le musée s'efforce de l'étudier, de l'exploiter et de le valoriser le mieux possible. Il en résulte un dispositif textuel particulièrement abouti et riche. Les niveaux d'information micro et

14 Les textes du plan macro-structurel renseignent sur la thématique de l'exposition et sa mise en scène: ce sont les textes introductifs ou conclusifs, les énoncés génériques des titres et des sous-titres. Ceux du plan micro-structurel sont plus directement mis en relation avec les objets : ils les nomment et peuvent les expliciter plus longuement. À ce propos, voir POLI, Marie-Sylvie. Le texte au musée: une approche sémiotique. Paris: L'Harmattan, 2002.

15 Les étiquettes autonymes "comportent un énoncé minimal composé de quelques mots, mais pas encore de phrases. Elles permettent au minimum de nommer l'objet et quelquefois d'en dire un peu plus, comme sa provenance, macro-structurels se distinguent ici aussi par leur apparence. Le niveau macro-structurel est celui des sous-titres écrits en grandes lettres sur les murs pour marquer les différents rassemblements thématiques. Le parcours se divise en sept groupes d'affiches: La Goulue: Aristide Bruant: Jane Avril: Café-concert: Des revues et des livres: Publicités: et Dernières affiches. Au niveau micro-structurel, chaque pièce de la collection est dotée d'une étiquette prédicative comportant le titre, la date, la technique et la provenance de l'œuvre (ce que contiennent les étiquettes autonymes), ainsi qu'un texte court mais élaboré au sujet du contenu de l'affiche et du contexte de sa création.

Les résultats de l'enquête qualitative ${ }^{16}$ nous apprennent que les visiteurs du musée d'Ixelles ont lu la plupart des textes des expositions EL CUBISMO et Lautrec, en général en entier. Ils les ont beaucoup appréciés et, à l'exception de quelques remarques d'ordre formel, les ont trouvés bien rédigés, d'une longueur satisfaisante et d'un niveau de langue très accessible. Presque tous les visiteurs ont eu le sentiment, en quittant le musée, d'avoir appris quelque chose, c'est-à-dire d'avoir acquis de nouvelles connaissances, mais aussi, et surtout, d'avoir interagi avec les œuvres d'art. L'étude révèle entre autres que plusieurs visiteurs ont lu avec beaucoup d'attention les cartels autonymes, qui leur paraissent indispensables pour appréhender une œuvre d'art. Les visiteurs préfèrent en général les panneaux muraux aux écrits mobiles. Souvent aussi, ils disent prendre le temps de lire avant ou après leur visite, soit pour acquérir des connaissances sur un thème ou un artiste, soit pour enrichir un savoir et prolonger la visite, séparant le

sa composition, sa date d'entrée dans les collections du musée». POLI, Ibid., p. 59.

16 "Les principales formes que peut prendre la recherche qualitative sont l'entrevue auprès de groupes cibles (focus group) et l'entrevue en profondeur. Ces méthodes ne visent pas à obtenir une mesure quantitative sur un sujet donné mais bien plutôt à comprendre des processus psychologiques, de saisir les motivations ou les perceptions des consommateurs à l'égard de produits ou de services. " LAPOINTE, Manon. Connaître ses visiteurs. Guide d'enquête par sondage. Montréal: Société des musées québécois, 2000, p. 15. 
temps de la lecture de celui de la contemplation. Ces résultats ont été obtenus par l'observation de visiteurs dans l'espace d'exposition et par entretiens semi-directifs à la sortie du musée. Des questions ouvertes ont permis de recueillir les éléments correspondant aux objectifs de l'enquête tout en offrant au visiteur le loisir de parler librement ou d'approfondir un point de son commentaire. Il était nécessaire de favoriser un climat de confiance pour faciliter la discussion et l'échange avec les visiteurs interviewés. Cet aspect de la méthodologie a une incidence non négligeable, car, grâce aux réactions spontanées et aux commentaires " déviants " des visiteurs interrogés, des constats inattendus et surprenants ont émergé. Il ressort de cette évaluation que certains types d'informations ont, plus que d'autres, des effets positifs sur la découverte des ouvres d'art par les visiteurs.

\section{Textes « intra-esthétiques»...}

Il est rapidement apparu au cours de l'enquête qu'un panneau de l'exposition EL CUBISMO connaissait un succès particulier auprès des visiteurs interrogés, à savoir le texte accompagnant l'œuvre de Juan Gris intitulée La fenêtre aux collines (ill. 1). Plusieurs répondants ont spontanément évoqué ce texte au cours des entretiens pour dire qu'il avait été un réel support face à l'œuvre et un soutien nécessaire et même agréable pour parvenir à la déchiffrer et à la comprendre. "Ce texte m'a aidé à entrer dans la peinture. » En quoi, exactement, le texte de La fenêtre aux collines se distingue-t-il des autres? Les propos tenus par les visiteurs se résument dans l'idée du texte qui " aide à regarder ": "Il y en a un très bien ici, celui qui explique ce tableau-là, celui-là est très bien parce qu'il apporte quelque chose, il explique, il donne des éléments d'explications de l'œuvre. " À l'issue de l'enquête réalisée au musée d'Ixelles, et de manière imprévue, nous avons été amenée à distinguer deux catégories d'écrits en fonction de la nature de leur contenu: les textes « intraesthétiques " et les textes "extra-esthétiques ". Plusieurs types de renseignements peuvent se retrouver dans un même texte, certains étant parfois très éloignés de la représentation picturale (par exemple des éléments biographiques). On parlera de texte "à dominance intra-esthétique" lorsque les explications d'ordre purement iconographique sont plus nombreuses et priment sur les autres.

Par quoi se définit un texte intra-esthétique? Tout d'abord, un texte ne saurait être intraesthétique s'il ne se réfère pas explicitement à une œuvre: il présente donc toutes les caractéristiques d'une étiquette autonyme: «Le texte de l'étiquette n'est pas un texte autonome, il se rapporte toujours à un expôt et lui donne du sens. C'est précisément ce rapport de dépendance qui intéresse le visiteur ${ }^{17}$." Le lien avec l'œuvre est d'autant plus fort qu'il se joue sur le mode sémantique et pas seulement spatial ou scénographique. Cependant, le texte intra-esthétique communique véritablement de l'information sur l'œuvre désignée. En cela, il correspond plus étroitement à la définition de l'étiquette prédicative, puisque celle-ci «comporte des phrases rédigées avec soin pour faire comprendre et faire connaître. C'est une aide à l'interprétation qui oriente la prise d'information. L'étiquette prédicative est souvent composée d'une ou de plusieurs phrases de base ${ }^{18}$." Au-delà de ces aspects, ce sont surtout les effets engendrés par son contenu qui lui confèrent le statut de texte intra-esthétique. La spécificité et fonction première de l'écrit intra-esthétique est de soutenir la contemplation de l'œuvre: il facilite et participe pleinement à la rencontre entre l'œuvre et le visiteur en développant un discours sur les éléments visibles et physiquement présents dans le tableau.

À travers une analyse iconographique, les mots amènent progressivement le visiteur à déchiffrer chacun des signes constitutifs de l'œuvre. Le sens des formes représentées, ou plutôt l'interprétation qu'en font les concepteurs de l'exposition, est transmis à travers l'étiquette pour soutenir l'exploration visuelle de l'œuvre. Ce texte s'est par ailleurs avéré particulièrement éclairant face à une œuvre

17 POLI, Le texte au musée..., op. cit., p. 93.

18 Ibid., p. 59. 


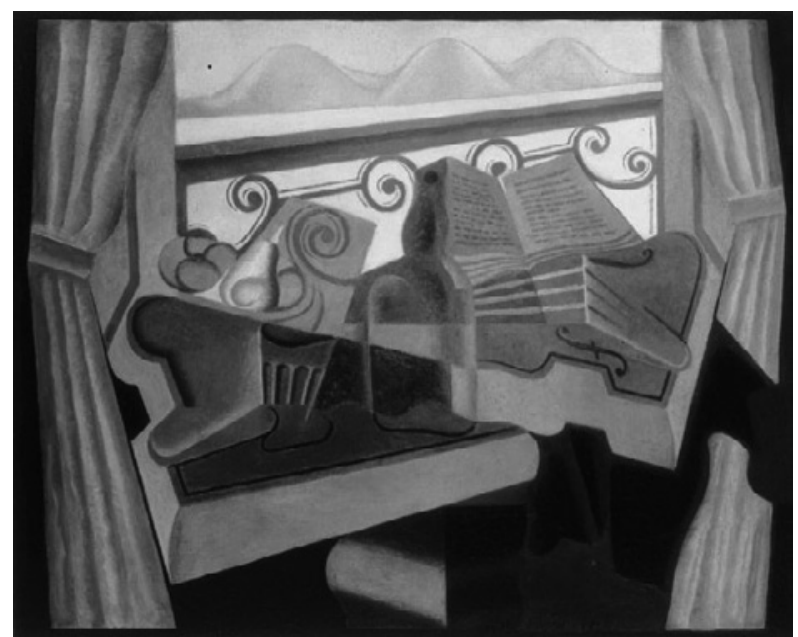

Illustration 1. Juan GRIS, La fenêtre aux collines, 1923.

Huile sur toile $(73 \times 92 \mathrm{~cm})$.

(C) Fundación Telefónica.

Dans La fenêtre aux collines, une rencontre intérieur-extérieur se produit, comme un jeu symbolique entre la notion d'intime et de publique [sic], entre art et vie, entre la poésie de la création et la poésie de la nature créatrice. Si la fenêtre ouverte fait allusion à l'impulsion vitale, à l'être au monde, le bouquet fait référence aux cinq sens : l'instrument de musique à l'ouïe, le vin et le fruit au goût et à l'odorat, la peau des fruits et les courbes des objets au toucher, la fenêtre et le livre ouverts à la vue. La sensualité n'était pas courante dans le cubisme "classique ", cependant Juan Gris la récupère dans la maturité de son œuvre.

Cette voie expressive devient un élément fort de son œuvre, comme si la quiétude de ce qui y est exposé était parcourue par une ultime expression, dans la couleur, la texture et le tracé: le tracé de la fenêtre se convertit en table, la table en violon, les lignes du violon sont les pages courbées du livre, le dos du livre ouvert rappelle la partie supérieure de la bouteille, de la bouteille sort une des spirales de la grille qui grimpe sur la poire et se fond avec le panier à fruits qui la soutient pour donner forme à un verre ou à une coupe dont le pied se confond avec la superficie de la table. Ces corrélations entre les formes et les figures sont des rimes plastiques ou rimes visuelles selon Juan Gris, comme en témoignent Raynal et Kahnweiler dans leurs écrits, Peinture et poésie. Un autre type de cubisme. 


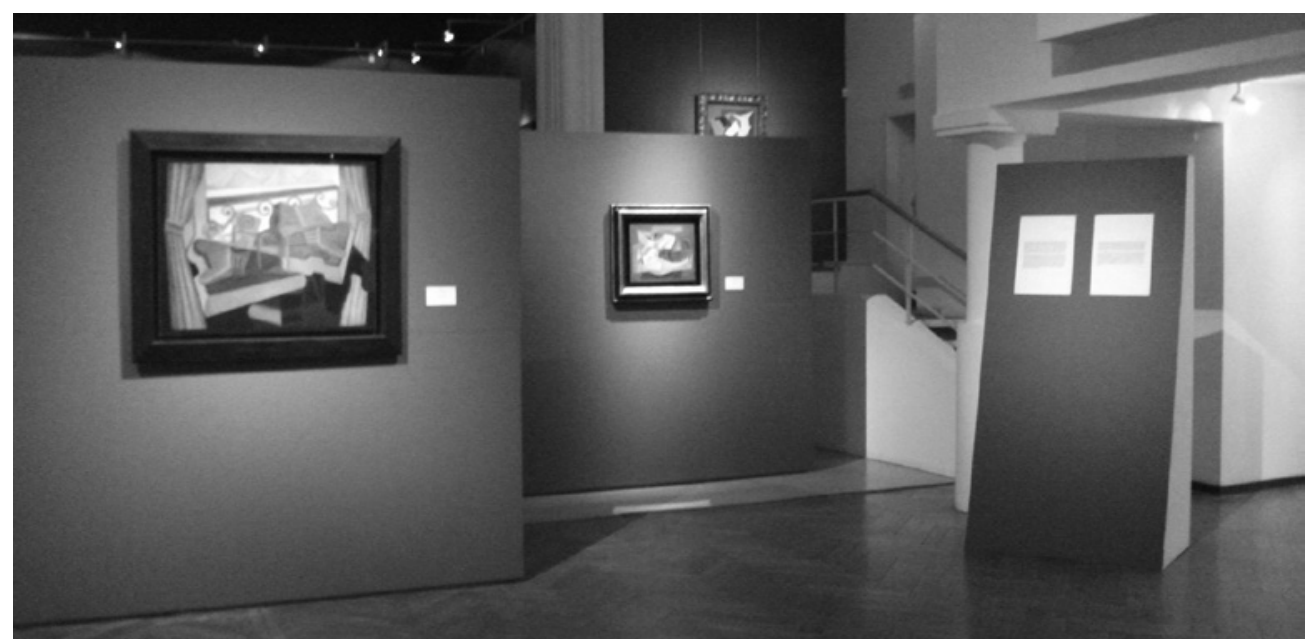

Il est rapidement apparu au cours de l'enquête qu'un panneau de l'exposition EL CUBISMO connaissait un succès particulier auprès des visiteurs interrogés, à savoir le texte accompagnant l'œuvre de Juan Gris intitulée La fenêtre aux collines (ill. 1).

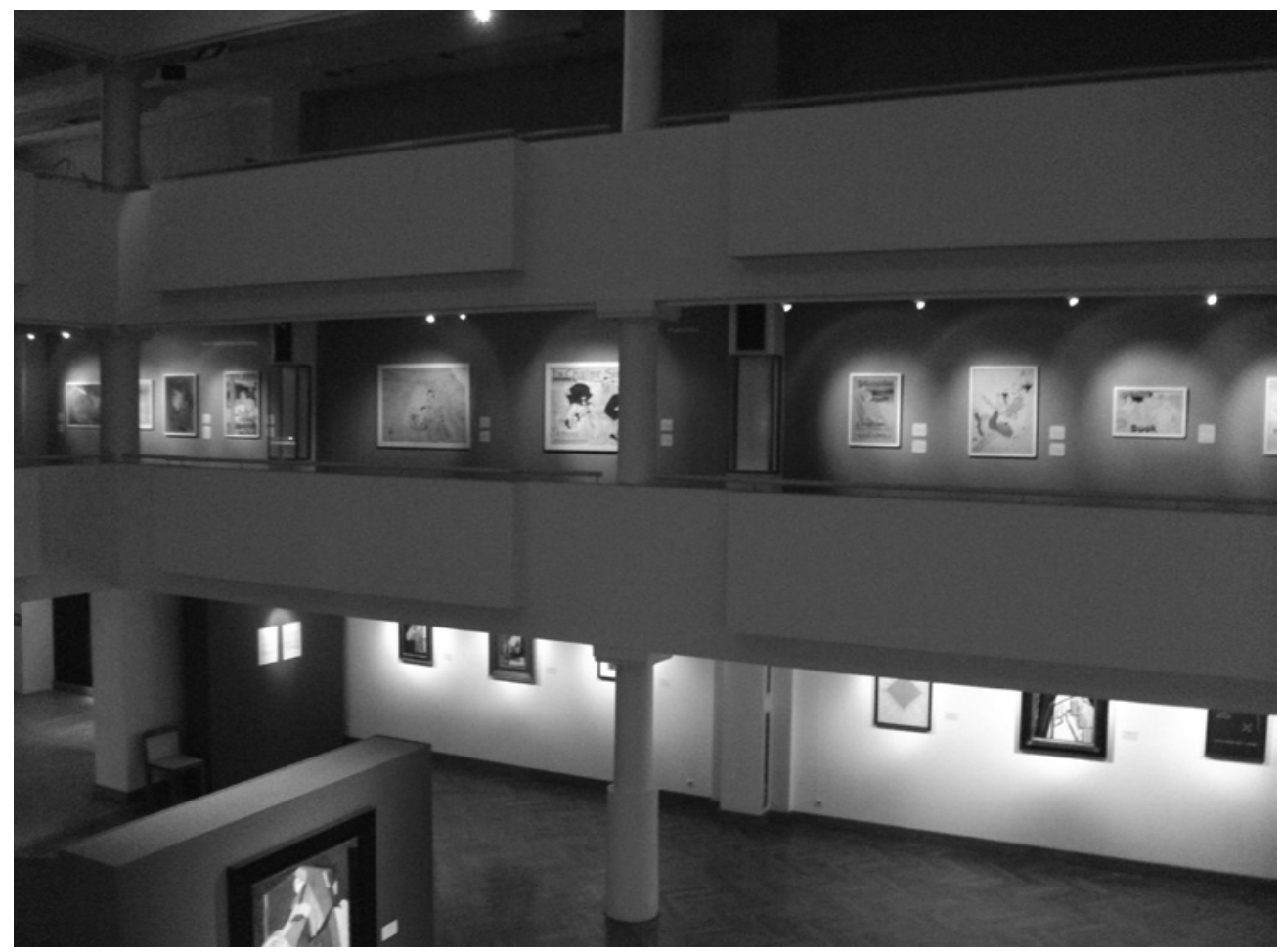

L'exposition des affiches de Lautrec, au premier étage, suit un parcours linéaire 


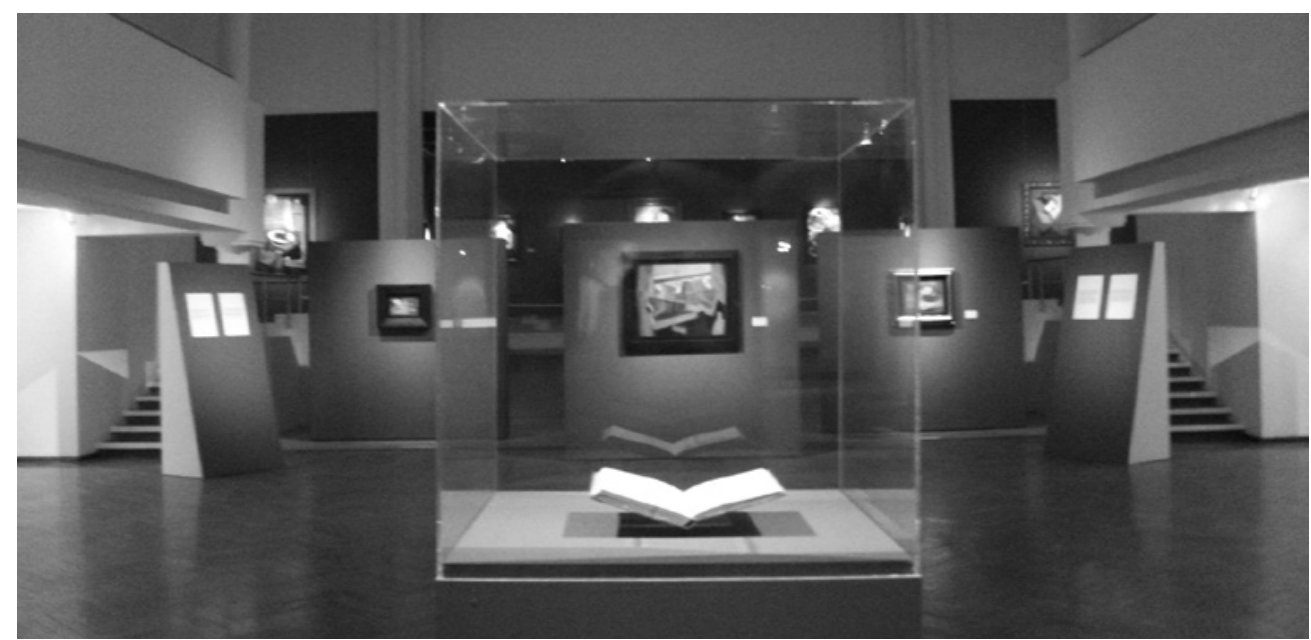

Les expositions temporaires du musée d'Ixelles se tiennent dans l'aile Morgan, un bâtiment rectangulaire de type halle, surplombé d'une verrière occultée. Il comprend un large espace au rez-de-chaussée, flanqué d'une scène, et deux galeries circulaires en balcon, la seconde étant depuis toujours fermée au public. L'exposition temporaire EL CUBISMO occupe le rez-de-chaussée, tandis que Lautrec occupe la première galerie. Il est intéressant de remarquer que cette architecture implique, pour la galerie circulaire, un parcours linéaire presque obligé, dans un sens ou dans un autre, mais forcément sans espace central de déambulation.

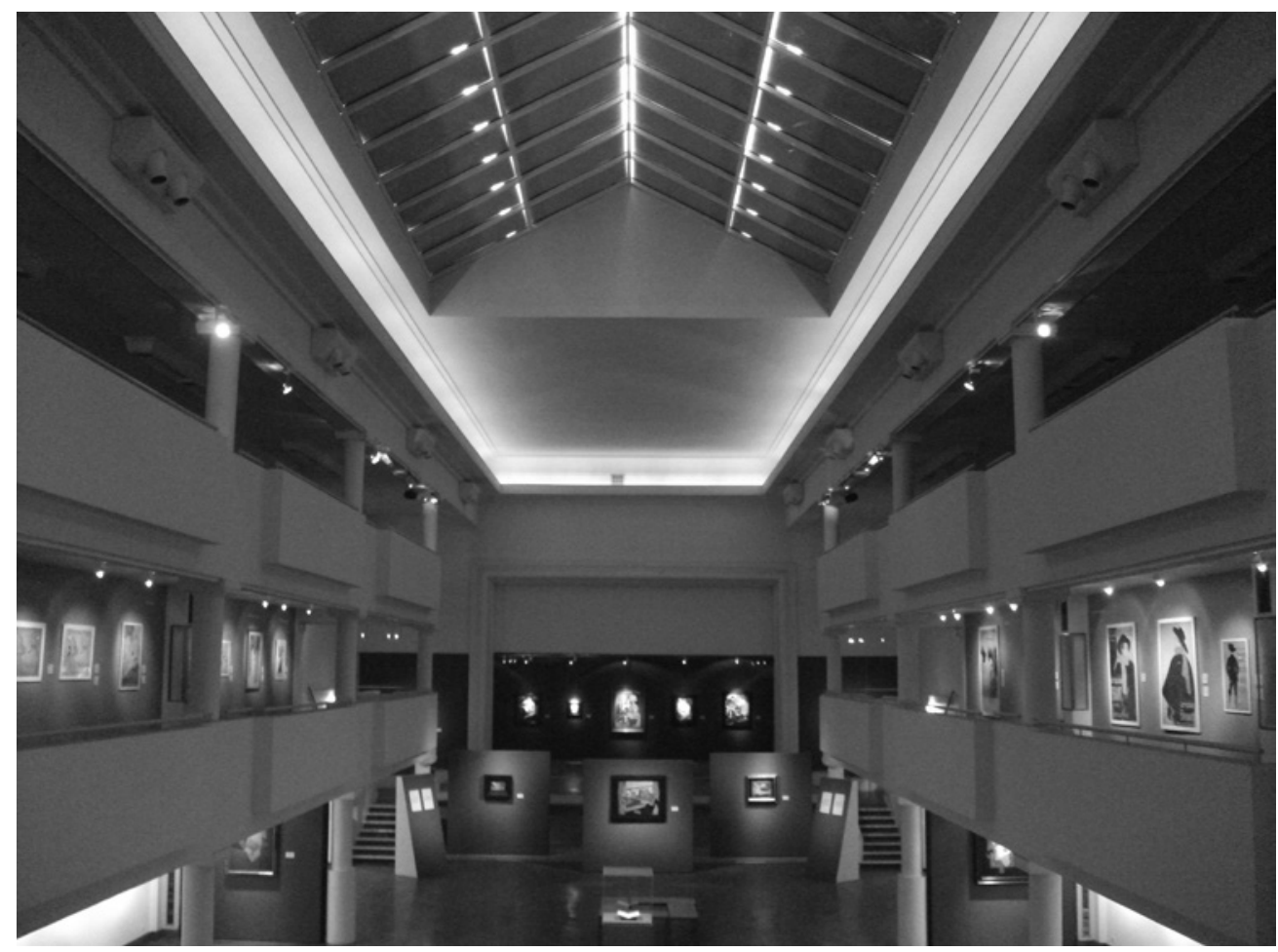

Images par Alice Chatzimanassis 
cubiste dont la représentation est déconstruite et la reconnaissance des éléments de la composition d'autant plus difficile: il pointe et décrit ce que le visiteur a sous les yeux.

Nous avons évoqué le lien presque intrinsèque qui existe entre l'œuvre et son cartel: ce lien est avant tout sémantique, mais il se constitue également à travers la qualité formelle du texte. MarieSylvie Poli explique que les stratégies linguistiques auxquelles recourt le scripteur (l'auteur du texte) pour traduire le discours scientifique en phrases lisibles et accessibles contribuent à instaurer « une relation de connivence entre le visiteur et l'objet " ${ }^{19}$. Parfois, les qualités de l'écriture " enrichissent à tel point le discours par l'entrée du poétique dans le texte [...] que l'attention du visiteur se porte alors sur le texte autant que sur l'objet ${ }^{20}$. On notera en l'occurrence l'usage, à deux reprises, de l'énumération, ce qui suscite un regard continu et progressif, de droite à gauche. L'énonciation, l'un après l'autre, de chaque signe plastique permet une lecture presque cursive du tableau de Juan Gris.

Les étiquettes prédicatives de l'exposition Lautrec peuvent aussi être qualifiées d'écrits intra-esthétiques. Ici, c'est le ton narratif qui, n'hésitant pas à verser dans l'anecdotique, a suscité le vif enthousiasme des visiteurs. Les étiquettes ont été appréciées précisément parce qu'elles racontent l'histoire de ce qui apparaît sur l'affiche. Plusieurs visiteurs ont dit s'être facilement laissés entraîner par le récit qu'elles relatent. Leur ton léger et leur format réduit ont souvent incité les visiteurs à les lire toutes, et au complet. "Les affiches, comme c'est représentatif d'une époque, c'est vrai que c'est intéressant de connaître les personnages sur l'affiche: il se trouve qu'elles étaient faites pour des personnes ou pour promouvoir certaines choses donc effectivement..." Pour chaque affiche, le texte explique le contexte dans lequel elle a été conçue, qui sont les personnages représentés et quelle place elle occupe dans la carrière de l'artiste. Lorsque le texte fait référence à des faits extérieurs, c'est parce que ceux-ci ont une incidence directe sur la représentation: le point de départ au développement du texte est toujours ce qui est visible dans l'œuvre. Le texte a donc le pouvoir de faire entrer le visiteur dans l'histoire des affiches, de le faire participer aux événements qui s'y jouent et de l'amener à saisir pleinement la personnalité de leur auteur.

\section{Là, là vraiment c'est tout à fait intéressant,} le texte ici est indispensable! Pour la bonne raison que Toulouse-Lautrec était un nabot, il était laid et tout et tout, mais il faisait partie de la haute noblesse, alors c'est amusant de voir qui il a choisi comme modèle $[. .$. Savoir que dans le coin d'une affiche, qui n'est pas sa meilleure d'ailleurs, on représente Guillaume II et que ça a fait une friction internationale, c'est dingue, on ne s'y attendait pas!

Les éléments anecdotiques ont d'ailleurs été perçus par les répondants comme nécessaires à la compréhension de l'affiche: ils se mêlent à l'analyse formelle et aux explications iconographiques et stylistiques. «Quand j'ai lu que le personnage était présenté de plusieurs manières différentes, notamment les profils, profil droit et profil gauche, c'est une chose à laquelle je n'avais pas prêté attention: si je n'avais pas eu le texte, je serais passé à côté. " Le franc succès des étiquettes prédicatives dans l'exposition Lautrec tient enfin à leur capacité à former, tout au long de l'exposition et au fil de chaque affiche, un tout cohérent tant du point de vue du contenu informatif que du langage. «Là par contre on a un fil comme ça pour suivre l'histoire, ça raconte une époque, c'est vraiment très chouette! Et ça explique vraiment la valeur des affiches. " $\mathrm{La}$ nature des informations et le type de discours utilisé, semblables sur toutes les étiquettes, permettent de rendre perceptible l'unité historique et stylistique des affiches de Lautrec. Cette harmonie est portée par la «fonction rhétorique macro-discursive " qui relie entre elles les étiquettes pour constituer un ensemble signifiant auquel le visiteur accède par la lecture: c'est elle qui « confère la cohérence de l'ensemble des textes et assure la cohésion discursive de l'exposition $"^{21}$.

21 Ibid., p. 60 


\section{...et textes «extra-esthétiques»}

Dans l'exposition Lautrec, les étiquettes se concentrent sur le contenu iconographique des affiches. De même, le texte de La fenêtre aux collines est en fin de compte le seul, sur un ensemble de onze panneaux, qui s'attarde véritablement sur une œuvre et en fournit une analyse. En cela, et par comparaison avec les autres écrits de l'exposition EL CUBISMO, il acquiert un statut tout à fait particulier ${ }^{22}$. Par opposition, on considère les dix autres textes comme des textes «à dominance extra-esthétique ». Ne se référant pas directement à la représentation picturale, le texte extra-esthétique agit comme un supplément, il apporte des connaissances qui dépassent l'œuvre. Des indications de ce type peuvent bien sûr être enrichissantes, mais elles n'appuient pas immédiatement l'appréhension visuelle de l'œuvre. Dans l'exposition EL CUBISMO, les quatre premiers textes qui se présentent au visiteur (numéros 1 à 4 sur le plan) sont conçus comme des entités complètement autonomes: «le panneau explicatif se suffit à lui-même et développe un discours autonome sur un thème sans se rattacher directement à un objet exposée ${ }^{23}$ ": ils dressent le cadre thématique général de l'exposition en évoquant l'histoire du cubisme, ses aspects stylistiques, la biographie de certains artistes, des citations de critiques d'art ou encore des éléments plus détaillés liés au parcours de Juan Gris. Ces quatre panneaux, indépendants entre eux du point de vue du contenu, sont par ailleurs physiquement séparés des œuvres: ils sont situés dans le vestibule qui précède la salle principale de l'exposition, sorte de sas à la jonction de l'accueil du musée, du vestiaire et des escaliers. Souvent d'ailleurs ces textes n'ont pas été remarqués par les visiteurs, en raison de leur emplacement. Quatre autres textes (5, 6, 10 et 11) sont des panneaux de section: un seul texte introduit une série de tableaux, par le biais des éléments historiques, contextuels ou biographiques qui n'expliquent pas de manière plus approfondie

22 La distinction relève exclusivement du contenu, car, du point de vue de l'aspect, ce texte présente les mêmes caractéristiques visuelles que les dix autres panneaux présents dans l'exposition. Les lettres noires sont inscrites sur du papier blanc de format A4 accroché directement sur la cimaise. l'une ou l'autre œuvre. Deux autres panneaux introduisent la section Géographie du Moderne, dont ils comportent le titre, et font référence à d'autres courants artistiques influencés par le cubisme. Les propos ne concernent donc plus les œuvres exposées ici. L'interrelation de tous ces textes avec les œuvres est finalement assez faible. Leur contenu évoque des éléments extérieurs aux œuvres en tant que telles.

On peut avancer, à la suite de ces quelques remarques, que les renseignements livrés par un texte à dominance extra-esthétique relèvent plutôt de la connaissance, tandis que ceux d'un texte intra-esthétique sont des explications à proprement dit: celles-ci permettent d'éclairer ce qui de prime abord pourrait sembler obscur. Il y a donc deux catégories d'informations différentes, mais qui peuvent être complémentaires. Nous n'affirmons pas ici la supériorité d'un type de texte par rapport à l'autre. Nous tentons simplement de souligner les divergences et de faire remarquer que des contenus de natures diverses produisent des effets variés chez les visiteurs quant à la compréhension d'une œuvre. Le texte extra-esthétique, réclamé par certains, satisfait la recherche de connaissances mais ne favorise pas forcément l'accès au sens purement plastique de l'œuvre. Par contre, le texte intra-esthétique a d'une certaine façon le pouvoir de placer tout le monde à égalité, puisque, sans exiger de connaissances ou de compétences préalables, il s'agit de ce que chacun peut voir, ce que chaque visiteur peut reconnaître dans le tableau. Destiné à soutenir la contemplation de l'œuvre, ce type de texte est susceptible de plaire à la majorité des visiteurs et peut-être particulièrement à ceux qui ne posséderaient pas d'entrée de jeu les références propres à l'histoire de l'art. Comment ne pas ici évoquer Bourdieu qui, dans L'Amour de l'art toujours, soulignait la demande de textes de la part des visiteurs "les moins cultivés " ? Contournant l'absence possible du " code social ${ }^{24}$ " nécessaire au déchiffrement

23 POLI, Marie-Sylvie. "Le parti-pris des mots dans l'étiquette: une approche linguistique ». Publics et Musées, $\mathrm{n}^{\circ} 1,1992$, p. 92.

24 BOURDIEU et DARBEL, L'amour de l'art..., op. cit. 
et à la compréhension d'une œuvre d'art, et qui relève du savoir et de la formation, le texte intra-esthétique livre véritablement des clés de lecture. Bourdieu nous dit en outre que les visiteurs pour qui l'art est moins familier se limitent souvent, et malgré eux, à la seule reconnaissance, dans une œuvre, des objets de la vie quotidienne (en d'autres termes les éléments "de leur monde") et qu'ils sont généralement incapables d'émettre des remarques d'ordre iconographique ou stylistique. Aussi le texte intra-esthétique représente-t-il une première étape vers une "exploration esthétique " et non plus exclusivement "sémantique" des œuvres d'art ${ }^{25}$.

\section{Conclusion}

La nature de l'information livrée, le sens et donc la fonction que l'on souhaite attribuer aux textes d'une exposition méritent en somme que l'on s'interroge. «L'étiquette a pour rôle d'aider le visiteur à interpréter ce qu'il voit, ce qu'il ressent. Pour cette raison, elle devrait d'abord fournir des informations qui stimulent l'observation de l'objet en aidant le visiteur à mieux le regarder ${ }^{26}$.» Dans les deux cas analysés ici, le texte de nature intra-esthétique a prouvé son efficacité. Sans pouvoir affirmer que, en tout temps, c'est ce type de texte qu'une exposition d'art doit favoriser, il n'est pas inutile de poser la question et d'y réfléchir. Le texte à dominance intra-esthétique a-t-il plus d'impact que les autres types de textes dans le processus de réception des œuvres d'art? Devrait-il, alors, être privilégié? Quelle doit être la nature des contenus textuels dans une exposition d'art et en fonction de quels objectifs ceux-ci sontils rédigés? La connaissance des visiteurs qui fréquentent l'institution et de leurs attentes peut être éclairante à certains égards. Le profil des visiteurs est parfois révélateur de leurs comportements et de leurs choix face aux dispositifs de médiation dans l'exposition. Dans le cadre de cette enquête, nous avons découvert

25 «Une exploration sémantique ou de contenu est plus rapide que l'exploration esthétique.» GOTTESDIENER, Évaluer l'exposition..., op. cit., p. 80.

26 DESJARDINS et JACOBI, «Les étiquettes dans les musées...". op.cit., p. 22. que, pour un certain nombre de visiteurs (12/36), «jouir des œuvres et en ressentir une émotion " est une motivation plus importante que le fait d'«acquérir des connaissances ", ce qui n'a pas empêché ces visiteurs de " consommer " largement les textes mis à leur disposition. Mais, pour ces personnes, la contemplation de l'œuvre doit primer sur la lecture du texte qui l'accompagne. Pour un nombre légèrement plus élevé de visiteurs (16/36), ces deux attentes sont aussi importantes l'une que l'autre. Par contre, l'acquisition de connaissances est une motivation plus importante que la jouissance de l'œuvre pour quelques personnes seulement (8/36). Cela explique peut-être pourquoi un type de texte a eu plus de succès qu'un autre. Le texte intra-esthétique répond parfaitement à la volonté première de jouir des œuvres. L'enquête réalisée au musée d'Ixelles nous a finalement rappelé que, dans une exposition d'art, la contemplation est, pour nombre de visiteurs, plus importante que l'acquisition de connaissances. Les textes devraient alors favoriser la rencontre esthétique, qui se complétera, éventuellement, par un apport de connaissances.

Affirmer que le texte accompagnant l'œuvre dans une exposition d'art devrait concerner explicitement et directement la représentation picturale pourra paraître d'une évidence écrasante. Rappelons cependant que, dans l'exposition EL CUBISMO, seul un texte parmi les onze répond à cette exigence. Plusieurs visiteurs interrogés au musée d'Ixelles ont d'ailleurs regretté qu'il n'y ait pas eu plusieurs textes de ce genre. Les musées de beaux-arts auraient-ils tendance à privilégier les connaissances historiques, biographiques et contextuelles au lieu de véritablement "donner à voir " les œuvres en présence? Il ne s'agit pas de militer pour un «nivellement du contenu par le bas " en faisant l'impasse sur tout exposé scientifique ou plus élaboré que la seule description. Les développements plus poussés et les interprétations savantes ont leur place dans l'exposition, mais peut-être pas sur les étiquettes prédicatives. Le moment où apparaît un texte dans le déroulement de la visite a semble-t-il son importance : face à l'œuvre, les renseignements iconographiques ou stylistiques seront 
toujours bienvenus, tandis qu'apparaissent,

ailleurs dans le parcours, des informations plus

générales et complémentaires, voire spéciali-

sées. C'est sans doute dans l'équilibre entre

les différents types et niveaux d'informations

à l'échelle de tout le "texte expographique ${ }^{27}$ "

que réside le pouvoir de l'écriture. 


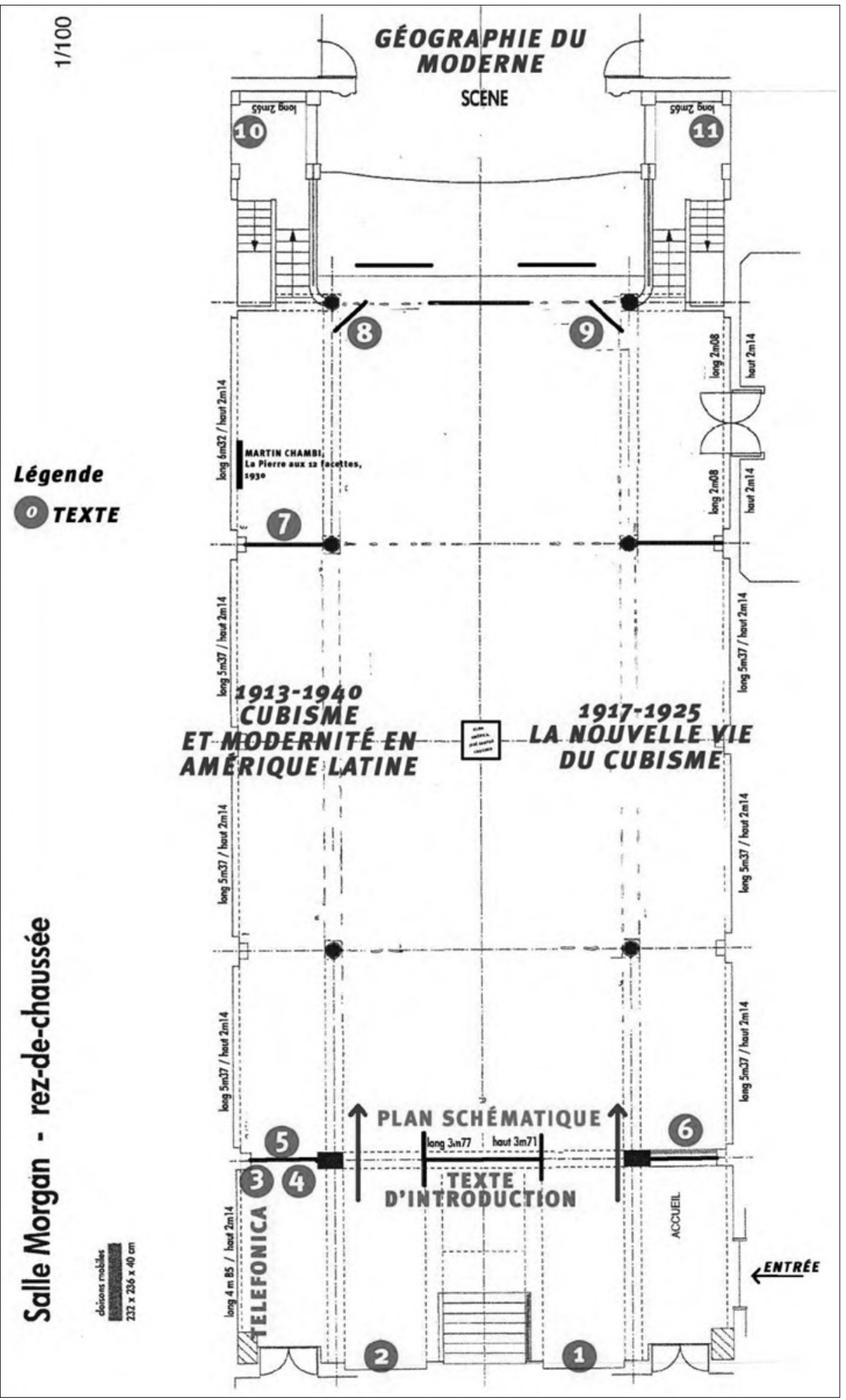

Annexe. Plan de l'exposition EL CUBISMO. Cubism and its Context. From the Fundación Telefónica Art Collection Plan: Camille Aubry, designer. 


\begin{abstract}
A survey was undertaken in April 2010 at the Museum of Ixelles, in Brussels. Interviews were conducted with visitors upon their leaving the site with the objective to identify and understand their modes of appropriation and their behaviours as regards the written notices used in two current exhibitions: EL CUBISMO. Cubism and its Context from the Fundación Telefónica Art Collection and Henri de Toulouse-Lautrec: La collection complète des affiches. If the importance of the text as an interpretation support in museums is now widely recognized, the qualitative survey has made it possible to further develop the reflection on the characteristics of the written tools when confronted to works of art, as well as their purpose in the visitors' contemplation and interpretation practices.
\end{abstract}

